\title{
Laparoscopic Pectopexy Vs Sacrohysteropexy/Sacrocolpopexy in Management of Pelvic Organ Prolapse: A Review of Literature
}

\author{
Dr. Nidhi Jain", Dr. Jyotsna Kamra, Dr. Shruthi Srinivas \\ Assistant Professor, Deptt of OBG, Maharaja Agarsein Medical College, Agroha, Hisar Haryana, India
}

DOI: $10.36348 /$ sijog.2020.v03i08.003

| Received: 22.06 .2020 | Accepted: 29.06 .2020 | Published: 11.08 .2020

*Corresponding author: Dr. Nidhi Jain

Abstract

Pelvic organ prolapse (POP) is a common disorder characterized by downward displacement of pelvic organs through vaginal wall. Women of advance age, multiparous, with history of previous gynaecological surgeries are at risk of developing POP. Various clinical features associated with POP are something coming of vagina, altered bladder habits or defecation problems. These features affects the quality of life of a women, thus timely management of POP is essential. It can be managed conservatively or surgically. Surgical approaches are hysterectomy, sacrohysteropexy, sacrocolpopexy and pectopexy. Sacrohysteropexy/ sacrocolpopexy are considered as the gold standard procedure of management of POP. However, recently, another procedure i.e. laparoscopic pectopexy is described as a new alternative procedure, which is equally effective and associated with lesser complications. Thus, authors hereby review the literature to compare laparoscopic pectopexy over Sacrohysteropexy/ sacrocolpopexy.

Keywords: laparoscopy, pectopexy, pelvic organ prolapse, sacrocolpopexy, sacrohysteropexy.

Copyright @ 2020: This is an open-access article distributed under the terms of the Creative Commons Attribution license which permits unrestricted use, distribution, and reproduction in any medium for non-commercial use (NonCommercial, or CC-BY-NC) provided the original author and source are credited.

\section{INTRODUCTION}

Pelvic organ prolapse (POP) is a common disorder seen in Asian women, characterized by downward displacement of pelvic organs (uterus/cervix/vault) through the vaginal wall. It affects almost $50 \%$ of parous women of age 50 years [1].

Various risk factors have been found to be associated with POP such as advancing age, multiparity, sexual activity, history of previous prolapse surgeries, history of urinary incontinence surgery, post hysterectomy [2]. After hysterectomy, incidence of vault prolapse is around 3.6/ 1000 women /year. This incidence further increases with increasing age [3].

Women with POP presents with various clinical features. One of the most common presenting complaints is something coming out of vagina. Symptoms due to vaginal protrusion like chronic pelvic pain, vaginal pressure, lower back pain or dyspareunia are commonly seen. Altered bladder habits are another chief complaint seen commonly which includes urinary retention, incontinence, irritative symptoms and obstructed voiding. Women with POP may complain of symptoms due to defecation dysfunction such as difficulty in passing stools, faecal urgency or incontinence [4]. These features affects the quality of life of a women, thus its management is essential and to be done timely.

Various approaches have been described for management of POP. It can be managed conservatively or surgically. Conservative management includes lifestyle advice, pelvic floor muscle training and use of mechanical devices. Surgical approach is done in case of third degree prolapse, hampering the quality of life. Various surgical approaches have been defined including hysterectomy, sacrohysteropexy, pectopexy and sacrocolpopexy. These procedures can be done by open technique or by minimally invasive techniques.

Sacrohysteropexy/ sacrocolpopexy are considered as the gold standard procedure of management of uterine/ vault prolapse [5]. However, recently, another procedure i.e. laparoscopic pectopexy is being described as a new alternative procedure, which is found to be equally effective and associated 


with far lesser complications than the
Sacrohysteropexy/ sacrocolpopexy.

\section{The authors thus hereby review the literature to compare laparoscopic pectopexy over} Sacrohysteropexy/ sacrocolpopexy.

\section{REVIEW OF LITERATURE}

Pelvic organ prolapse is one of the most common indication for which gynaecological surgeries are being done. Many different procedures via different route have been described over period of time. Vaginal hysterectomy is one of the most traditional methods being performed for uterine prolapse in old age women. However, in young women with uterine prolapse, conservative procedures are done that includes sacrohysteropexy and pectopexy. Routes via which surgery is done, includes open abdominal method, laparoscopic approach or robotic technique. In old age women with vault prolapse, sacrocolpopexy or pectopexy can be done.

\section{Sacrocolpopexy / sacrohysteropexy}

Sacrohysteropexy/ sacrocolpopexy are a wellknown technique which is considered as gold standard technique for management of pelvic organ prolapse [4]. In this technique, mesh is applied below the level of sacral promontory and attached to the uterine isthmus or apex of vault, thereby reconstituting the physiological axis of vagina. However, some surgeons prefer to put mesh at anterior longitudinal ligament over second sacral vertebrae (S2). This procedure can be done via open technique (abdominal approach), laparoscopically or via robotic method. Although sacropexy is considered as the most effective procedure for prolapse management, but it is also associated with several Intraoperative and post-operative complications. Intraoperative complications include visceral injuries such as risk of damage to sigmoid colon and ureter. Another major intra operative complication is life threatening haemorrhage due to injury to presacral vessels. Post-operative complications include gastrointestinal complications, defecation disorders and urinary complaints [6]. Since in this procedure, mesh is placed between sacrum and vagina, so it leads to pelvic outlet obstruction causing the defecation disorders. Defecation problems can also occur due to adhesions formation or due to trauma to hypogastric nerve during the procedure [6-10].

In 2004, Nygaard IE et al. [7] conducted a study in which literature was searched on MEDLINE to summarize published data on abdominal sacrocolpopexy and to highlight area about which data are lacking. It was found that the success rate, defined as lack of prolapse postoperatively, ranged from $58 \%$ $100 \%$ and success rate in terms of lack of apical prolapse was $78 \%$ to $100 \%$. The median reoperation rate for pelvic organ prolapse was $4.4 \%$ and for stress urinary incontinence were $4.9 \%$. Overall rate of mesh erosion was $3.4 \%$. Risk of small bowel obstruction requiring surgery postoperatively was found to be 1.1 $\%$. This it was concluded that sacrocolpopexy is a reliable procedure for management of POP, but it is associated with risk of Resurgery for recurrence of prolapse or incontinence or other gastrointestinal complications, for which patients should always be counselled.

In a study by Huebner $\mathrm{M}$ et al. [11] in 2009, detailed description of abdominal sacrocolpopexy and retrospective evaluation of the outcomes was provided. 78 women underwent surgery of which 53 women participated in follow up. In term of apical prolapse, none of the patient had recurrence, thus $100 \%$ success rate was seen. In term of anterior wall prolapse, $17 \%$ had stage II anterior wall prolapse but $69.8 \%$ of them were asymptomatic. Regarding the posterior compartment, $38 \%$ women had posterior wall prolapse, however $86.8 \%$ of them were asymptomatic. Nine patients among them required Resurgery.

In 2014, a study was done by Sarlos D et al. [12], to evaluate the long term follow up of laparoscopic sacrocolpopexy. 101 patients were included in the study and their follow up was done at 5 years. 11 patients were found to have anatomical recurrence, of which 6 women had anterior wall prolapse, 4 had posterior wall and 1 woman had apical prolapse. The total reoperation rate was $3.5 \%$. Mesh erosion was seen in 2 cases, and in both cases, erosion occurred into the bladder.

In a study by Whitehead WE et al. [9], gastrointestinal complications following abdominal sacrocolpopexy were assessed. It was found that, out of 322 women, $18 \%$ reported nausea, emesis, bloating or ileus during hospitalization period postoperatively and $9.8 \%$ reported these same complaints at 6 weeks. Nineteen women $(5.9 \%)$ had possible ileus or small bowel obstruction of which 4 women were reoperated, 11 of them were readmitted for medical management and 4 of them had prolonged hospitalization. Old age was found to be a significant risk factor for ileus or small bowel obstruction. Thus it was concluded that patient should always be counselled regarding Gastrointestinal complications before undergoing the procedure.

In young women also, this procedure is done commonly. Sumera Tahir et al. [13] did a study to see outcome of Abdominal Sacrohysteropexy in Young Women with Uterovaginal Prolapse. The study was carried over a period of one year. 12 women with uterovaginal prolapse wishing to retain their uterus underwent Sacrohysteropexy with Prolene Mesh attached to uterine isthmus and to the anterior longitudinal ligament of the first or second sacral vertebra in a tension free fashion. Subjective and objective cure of uterine prolapse and operative and 
postoperative complications were noted. The mean age of patient was 30 years (range 16-40 years). The mean follow up period was 12 months. No women developed intra and postoperative complications. No single case of mesh erosion was detected. Success rate was $83.3 \%$. Recurrent prolapse was recorded in $2(16.7 \%)$ women who was symptomatic and required repeat surgical treatment. Thus it was concluded that Sacrohysteropexy is effective and safe procedure in women with uterovaginal prolapse who want to preserve their uteri.

Hence, sacrocolpopexy/ sacrohysteropexy are an effective method of managing the pelvic organ prolapse but it is associated with several Intraoperative and postoperative complications. To overcome these complications, Banerjee \& Noe [14] has described another procedure, laparoscopic pectopexy for repair of apical prolapse, in which bilateral ileopectineal ligaments are used for mesh fixation.

\section{Laparoscopic Pectopexy}

Laparoscopic pectopexy is effective, safe and comfortable alternative method for management of pelvic organ prolapse. It was described by Banerjee \& Noe [14] in 2011, especially in obese women.

In this procedure, a transverse mesh is fixed on lateral part of ileopectineal ligament via non absorbable suture. Iliopectineal ligament is defined as extension of lacunar ligament which runs on pectineal line of pubic bone as shown in figure 1. It is stronger than sacrospinous ligament and arcus tendineus of pelvic fascia. Since, the ligament is attached on second sacral vertebrae, so anchoring the vaginal tissue on it, helps in maintaining the physiological axis. Secondly, mesh follows the normal anatomical structures i.e. round ligament, broad ligament and iliopectineal ligament without disturbing the pelvic outlet. Also, procedure is done in the wide operating field, which is far distant from ureter, bowel and vessels, hence minimising the risk of injuries to these vital structures.

In 2011, Banerjee C et al. [14] described laparoscopic pectopexy as a new technique for prolapse surgery in obese patients. They successfully performed the procedure in 12 patients without any complications. Lesser morbidity and lesser operative time were found during laparoscopic pectopexy. They found that performing conventional technique in very obese patients is sometimes restricted due to difficulty in performing the procedure. Hence it was concluded that laparoscopic pectopexy provides a stable and durable repair.

In 2015, Noe KG et al. [15] conducted a pilot study to compare outcome of laparoscopic sacral colpocervicopexy and laparoscopic pectopexy. They randomly assigned the patients to two treatment group: 44 in pectopexy and 41 in sacropexy. All defects were managed at same time. Follow up examination was done over period of 12 to 37 months (mean follow up 20.67 months). A clear difference was found regarding de no defecation disorders $(0 \%$ in pectopexy, $19.5 \%$ in sacropexy). The incidence of de novo urinary incontinence and rectocoele were similar in both the groups. The apical descent rate was $2.3 \%$ in pectopexy and $9.8 \%$ in sacropexy group, however difference was statistically insignificant. Since, pectopexy does not reduce the pelvic space, so defecation disorders were seen in none of the patients. Hence it was concluded that laparoscopic pectopexy is a novel method of vaginal prolapse management that offers clear practical advantage over laparoscopic sacropexy.

In 2017, a study was done by Ahmet Kale et al. [16], in which authors shared their experience about laparoscopy pectopexy and its feasibility. Surgery was done in seven patients with apical prolapse. No intra operative and postoperative complications were found. During 6 month follow up period, de novo apical prolapse/urinary complaints/ bowel complaints were not seen in any of the patients. Thus it was concluded that laparoscopic pectopexy is a feasible, safe and comfortable alternative to sacrocolpopexy and also, laparoscopic pectopexy may increase a surgeon's technical perspective for management of apical prolapse.

In 2018, Alper Biler et al. [17] did a study to compare perioperative complications and short term outcomes of abdominal sacrocolpopexy, laparoscopic sacrocolpopexy and laparoscopic pectopexy for apical prolapse. A total of 68 abdominal surgeries (44 abdominal sacrocolpopexy and 24 sacrohysteropexy), 14 laparoscopic surgeries (10 laparoscopic sacrocolpopexy, 4 laparoscopic hysteropexy) and 28 laparoscopic pectopexy were done. Baseline and Intraoperative variables were same in all three groups. The mean operating time was found significantly shorter in laparoscopic pectopexy group (74.9 minutes). At six month follow up, there had been no occurrences of de novo apical prolapse, anterior/ lateral defect cystocoeles, and rectocoele or mesh erosion in any group. However, one case of dyspareunia, four cases of de novo urgency, two de novo SUI cases was seen in abdominal sacrocolpopexy group. One case of de novo persistent constipation, one case of de novo urgency, one case of de novo SUI occurred in laparoscopic sacrocolpopexy group. Two cases of de novo urgency and one case of SUI were seen in laparoscopic pectopexy group. Hence, it was concluded that abdominal sacrocolpopexy, laparoscopic sacrocolpopexy and laparoscopic pectopexy have comparable perioperative complications and short term anatomical and subjective outcomes. Although complications rate were not significantly different between three groups, but laparoscopic sacrocolpopexy and laparoscopic pectopexy were found to have less morbidity. Moreover, laparoscopic pectopexy had 
shorter operating time, so it can be used as a preferred method by surgeon over sacrocolpopexy.

Hence from review of literature it was found that laparoscopic pectopexy offers several practical advantages over abdominal/ laparoscopic sacrocolpopexy, which are as follows:

- It provides safe surgical field, so no risk of Intraoperative injury to ureter, bowel and vessels.
- It provides wide area of surgery, thus helpful in operating during complicated conditions.

- Since, it does not reduce the pelvic space, thus there is no risk of defecations disorders postoperatively.

- Since iliopectineal ligament is a strong ligament, so there is minimal risk of recurrence of apical prolapse postoperatively.

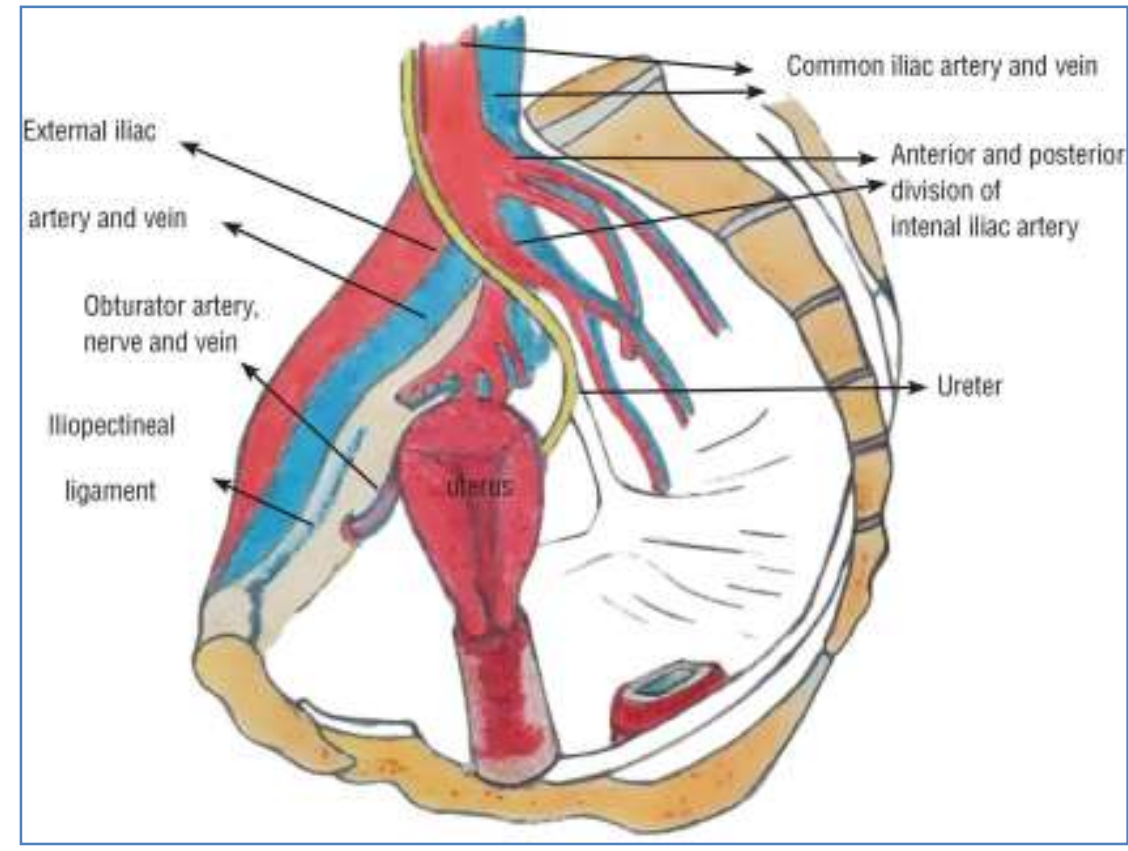

Fig-1: Anatomical Details of Iliopectineal Ligament

\section{CONCLUSION}

Authors hereby conclude that laparoscopic pectopexy is a safe, effective and feasible alternative approach in management of pelvic organ prolapse. Moreover, it is associated with minimal intraoperative and postoperative complications, so should be preferred over sacrohysteropexy/ sacrocolpopexy in management of pelvic organ prolapse.

\section{REFERENCES}

1. Subak, L. L., Waetjen, L. E., Van Den Eeden, S., Thom, D. H., Vittinghoff, E., \& Brown, J. S. (2001). Cost of pelvic organ prolapse surgery in the United States. Obstetrics \& Gynecology, 98(4), 646-651.

2. Dällenbach, P., Kaelin-Gambirasio, I., Jacob, S., Dubuisson, J. B., \& Boulvain, M. (2008). Incidence rate and risk factors for vaginal vault prolapse repair after hysterectomy. International Urogynecology Journal, 19(12), 1623-1629.

3. Mant, J., Painter, R., \& Vessey, M. (1997). Epidemiology of genital prolapse: observations from the Oxford Family Planning Association Study. BJOG: An International Journal of Obstetrics \& Gynaecology, 104(5), 579-585.
4. Beer, M., \& Kuhn, A. (2005). Surgical techniques for vault prolapse: a review of the literature. European Journal of Obstetrics \& Gynecology and Reproductive Biology, 119(2), 144-155.

5. Maher, C., Feiner, B., Baessler, K., \& Schmid, C. (2013). Surgical management of pelvic organ prolapse in women. Cochrane database of systematic reviews, (4).

6. Akladios, C. Y., Dautun, D., Saussine, C., Baldauf, J. J., Mathelin, C., \& Wattiez, A. (2010). Laparoscopic sacrocolpopexy for female genital organ prolapse: establishment of a learning curve. European Journal of Obstetrics \& Gynecology and Reproductive Biology, 149(2), 218-221.

7. Nygaard, I. E., McCreery, R., Brubaker, L., Connolly, A., Cundiff, G., Weber, A. M., \& Zyczynski, H. (2004). Abdominal sacrocolpopexy: a comprehensive review. Obstetrics \& Gynecology, 104(4), 805-823.

8. Sarlos, D., Brandner, S., Kots, L., Gygax, N., \& Schaer, G. (2008). Laparoscopic sacrocolpopexy for uterine and post-hysterectomy prolapse: anatomical results, quality of life and perioperative outcome - a prospective study with 101 
cases. International

Journal, 19(10), 1415-1422.

Urogynecology

9. Whitehead, W. E., Bradley, C. S., Brown, M. B., Brubaker, L., Gutman, R. E., Varner, R. E., ... \& Network, P. F. (2007). Gastrointestinal complications following abdominal sacrocolpopexy for advanced pelvic organ prolapse. American journal of obstetrics and gynecology, 197(1), 78-e1.

10. Kumar, S., Malhotra, N., Chumber, S., Gupta, P., Aruna, J., Roy, K. K., \& Sharma, J. B. (2007). Control of presacral venous bleeding, using thumbtacks. Archives of gynecology and obstetrics, 276(4), 385-386.

11. Huebner, M., Krzonkalla, M., \& Tunn, R. (2009). Abdominal sacrocolpopexy-standardized surgical technique, perioperative management and outcome in women with posthysterectomy vaginal vault prolapse. Gynäkologisch-geburtshilfliche Rundschau, 49(4), 308-314.

12. Sarlos, D., Kots, L., Ryu, G., \& Schaer, G. (2014). Long-term follow-up of laparoscopic sacrocolpopexy. International urogynecology journal, 25(9), 1207-1212.

13. Barranger, E., Fritel, X., \& Pigne, A. (2003). Abdominal sacrohysteropexy in young women with uterovaginal prolapse: long-term follow- up. American journal of obstetrics and gynecology, 189(5), 1245-1250.

14. Banerjee, C., \& Noé, K. G. (2011). Laparoscopic pectopexy: a new technique of prolapse surgery for obese patients. Archives of gynecology and obstetrics, 284(3), 631-635.

15. Noé, K. G., Schiermeier, S., Alkatout, I., \& Anapolski, M. (2015). Laparoscopic pectopexy: a prospective, randomized, comparative clinical trial of standard laparoscopic sacral colpocervicopexy with the new laparoscopic pectopexyPostoperative results and intermediate-term followup in a pilot study. Journal of endourology, 29(2), 210-215.

16. Kale, A., Biler, A., Terzi, H., Usta, T., \& Kale, E. (2017). Laparoscopic pectopexy: initial experience of single center with a new technique for apical prolapse surgery. International braz j urol, 43(5), 903-909.

17. Biler, A., Ertas, I. E., Tosun, G., Hortu, I., Turkay, U., Gultekin, O. E., \& Igci, G. (2018). Perioperative complications and short-term outcomes of abdominal sacrocolpopexy, laparoscopic sacrocolpopexy, and laparoscopic pectopexy for apical prolapse. International braz $j$ urol, 44(5), 996-1004. 